Article

\title{
Pharmacokinetic Comparisons of Benzoylmesaconine in Rats Using Ultra-Performance Liquid Chromatography-Tandem Mass Spectrometry after Administration of Pure Benzoylmesaconine and Wutou Decoction
}

\author{
Pei-Min Dai ${ }^{1,2}$, Ying Wang ${ }^{1,2}$, Ling Ye ${ }^{1}$, Shan Zeng ${ }^{1}$, Zhi-Jie Zheng ${ }^{1}$, Qiang Li ${ }^{1}$, Lin-Liu Lu ${ }^{1,2}$ \\ and Zhong-Qiu Liu ${ }^{1,2, *}$
}

1 Department of Pharmaceutics, School of Pharmaceutical Sciences, Southern Medical University, Guangzhou 510515, Guangdong, China; E-Mails: daipeimin1989@163.com (P.-M.D.);

IITCM_Wangy@126.com (Y.W.); lotus623@126.com (L.Y.); zengshan_jessica@126.com (S.Z.); zhijiezheng@126.com (Z.-J.Z.); liqiangsmu@163.com (Q.L.); iitcm_lu@126.com (L.-L.L.)

2 International Institute for Translational Chinese Medicine, Guangzhou University of Chinese Medicine, Guangzhou 510006, Guangdong, China

* Author to whom correspondence should be addressed; E-Mail: liuzq@gzucm.edu.cn; Tel.: +86-20-39358061; Fax: +86-20-39358071.

External Editor: Derek J. McPhee

Received: 11 September 2014; in revised form: 8 October 2014 / Accepted: 8 October 2014 / Published: 17 October 2014

\begin{abstract}
Wutou decoction is widely used in China because of its therapeutic effect on rheumatoid arthritis. Benzoylmesaconine (BMA), the most abundant component of Wutou decoction, was used as the marker compound for the pharmacokinetic study of Wutou decoction. The aim of the present study was to compare the pharmacokinetics of BMA in rats after oral administration of pure BMA and Wutou decoction. Pure BMA $(5 \mathrm{mg} / \mathrm{kg})$ and Wutou decoction $(0.54 \mathrm{~g} / \mathrm{kg}$, equivalent to $5 \mathrm{mg} / \mathrm{kg}$ BMA) were orally administered to rats with blood samples collected over $10 \mathrm{~h}$. Quantification of BMA in rat plasma was achieved using sensitive and validated ultra-performance liquid chromatography-tandem mass spectrometry (UPLC-MS/MS). Specifically, the half-life $\left(T_{1 / 2}\right)$ and mean residence time values of pure BMA were $228.3 \pm 117.0 \mathrm{~min}$ and $155.0 \pm 33.2 \mathrm{~min}$, respectively, whereas those of BMA in Wutou decoction were decreased to $61.8 \pm 35.1 \mathrm{~min}$ and $55.8 \pm 16.4 \mathrm{~min}$, respectively. The area under the curve (AUC) of BMA after administration of Wutou decoction was significantly decreased (five-fold) compared with that of pure BMA. The
\end{abstract}


results indicate that the elimination of BMA in rats after the administration of Wutou decoction was significantly faster compared with that of pure BMA.

Keywords: benzoylmesaconine; Wutou decoction; pharmacokinetics; UPLC-MS/MS

\section{Introduction}

Herbal medicine is mostly administered in combination based on the principle of traditional Chinese Medicine to achieve effect optimization or toxicity reduction. It is generally recognized that the combined use of prescriptions have a greater impact on the therapeutic effects. Pharmacokinetic study, a useful method to predict the in vivo process, has been used to elucidate the different processes between pure chemicals and multiple-ingredient prescriptions; therefore, it is important to conduct pharmacokinetic studies to evaluate the rationality of the traditional prescriptions.

Wutou decoction, a well-known traditional prescription, comprises Radix Aconiti (derived from the dried root of Aconitum carmichaeli Debx.), Herba Ephedrae (derived from the stem of Ephedra sinica Stapf.), Radix Paeoniae Alba (derived from the dried root of Paeonia lactiflora Pall.), Radix Astragali (derived from the dried root of Astragalus membranaceus) and Radix Glycyrrhizae (derived from the dried root of Glycyrrhiza uralensis Fisch). The decoction has been used for hundreds of years in China for rheumatoid arthritis (RA) treatment.

To date, many studies have investigated the effects of Wutou decoction in patients with RA. Radix Aconiti, a naturally occurring product, is considered a prominent component of Wutou decoction because of its therapeutic effects against RA [1,2]. However, Radix Aconiti is a toxic herb that can cause serious cardiac poisoning [3-6]. Previous studies revealed a 58\% incidence rate of toxic reactions among 188 patients who had taken aconitum [7-9]. Aconitum alkaloids are the effective chemicals of Radix Aconiti. Notably, the high levels of toxicity of Radix Aconiti are derived from diester aconitum alkaloids, including aconitine, mesaconitine (MA) and hypaconitine, which can be hydrolyzed to benzoylaconine, benzoylmesaconine (BMA) and benzoylhypaconine, respectively. The MA hydrolysate BMA demonstrates lower toxicity [7], higher abundance and a better pharmacological effect than the other two monoester diterpenoid alkaloids in Wutou decoction [10]. This study selected BMA as a marker compound for the pharmacokinetic study of Wutou decoction.

BMA exhibits biological effects, including analgesic [11,12], antiviral and antifungal activities [13]. It can also stimulate cytokine secretion [14]. Although BMA is less toxic than diester diterpene alkaloids [10], excessive intake of BMA still causes toxic reactions [15]. Hence, several pharmacokinetic studies have been performed to understand the in vivo process of BMA by using the LC-MS method [16-20]. For instance, some researchers [16] found that BMA demonstrates fast absorption and elimination in vivo after the administration of pure BMA ( $\left.T_{1 / 2} \beta=407 \pm 180 \mathrm{~min}\right)$. Others [17] verified that BMA has short $T_{\max }(36.17 \pm 1.72 \mathrm{~min})$ compared with the other two monoester aconitum alkaloids after intravenous drop infusion of "SHEN-FU" injectable powder. Recently, research on the pharmacokinetics of BMA was conducted in combination with other herbs or components, such as Dahuang-Fuzi decoction and Radix et Rhizoma Rhei [18,19]. The pharmacokinetic parameters $\left(\mathrm{C}_{\max }, \mathrm{AUC}\right)$ of BMA were remarkably reduced; $T_{1 / 2}$ and mean residence 
time (MRT) were delayed with oral administration of Dahuang-Fuzi decoction [18]. The additional studies demonstrated that co-administration with Rhizoma Zingiberis could reduce the $\mathrm{C}_{\max }$ of BMA with the increased $T_{1 / 2}, T_{\max }$ and AUC [19]. The co-administration suggests that Rhizoma Zingiberis or other ingredients in Dahuang-Fuzi decoction could not accelerate the elimination of BMA, therefore having little influence on the toxicity reduction.

Although the above studies focused on the pharmacokinetics of BMA [16-20], little is known about the pharmacokinetics after the administration of Wutou decoction and pure BMA. Since Wutou decoction has complex ingredients, herb-herb interactions may exert an influence on the efficacy of Chinese medicine; therefore, this study aims to compare the pharmacokinetics of BMA after oral administration of pure BMA and Wutou decoction to rats using a rapid, sensitive and reliable UPLC-MS/MS method. The information obtained might be useful for understanding different herb-herb interactions between BMA and other ingredients, which can be used as a reference for the clinical administration of Wutou decoction.

\section{Results and Discussion}

\subsection{Validation of UPLC-MS/MS Methods}

An Acquity UPLC BEH C18 column was selected for the chromatographic separation of BMA (Figure 1A) and testosterone (Figure 1B), because of its good peak shapes and acceptable retention times. A 2-mM ammonium acetate and acetonitrile solution led to better peak shapes compared to other mobile phases. Testosterone was selected as an internal standard, because of its similar chromatographic behavior and complete separation with BMA.

Figure 1. Chemical structures of benzoylmesaconine (BMA) (A) and the internal standard, testosterone $(\mathbf{B})$.

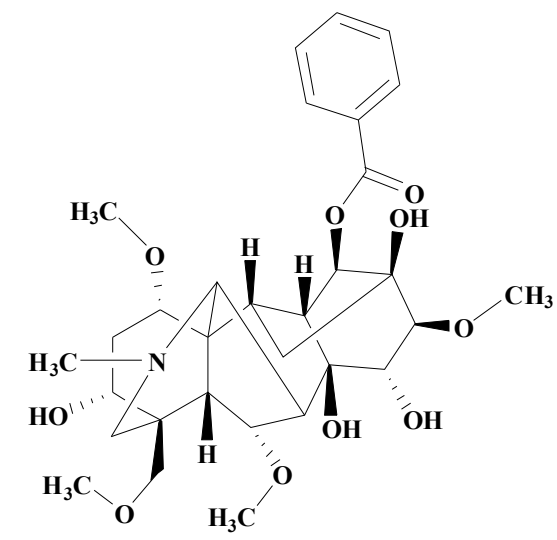

(A)

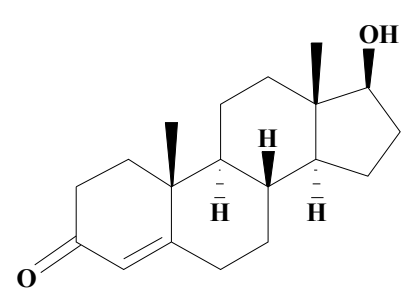

(B)

The protein precipitation method developed in this study for the preparation of plasma samples was more simple and cost-effective compared with those developed in previous studies [17,21,22]. Several organic solvents, such as methanol, acetonitrile and ethyl acetate, were investigated for extraction. The results showed that BMA and testosterone could not be completely extracted simultaneously by acetonitrile, ethyl acetate and ethanol. Methanol was finally selected for sample preparation, because 
of its relatively high extraction recovery. Methanol-water (1:1), instead of the mobile phase, has been used to reconstitute the residue, because of the better peak shape. Therefore, a one-step process of protein precipitation with methanol that exhibited acceptable recovery was selected. This process was time saving and helpful to the stability of plasma samples.

The concentrations of BMA in the plasma samples were too low to be determined by UPLC-UV. Thus, an UPLC-MS/MS method with higher sensitivity than UPLC-UV was developed in this study. The MS and MS/MS daughter scan spectrograms of BMA and testosterone in the ESI source are shown in Figures 2 and 3, respectively.

Figure 2. Full-scan (A) and MS/MS daughter scan (B) spectrograms of BMA.
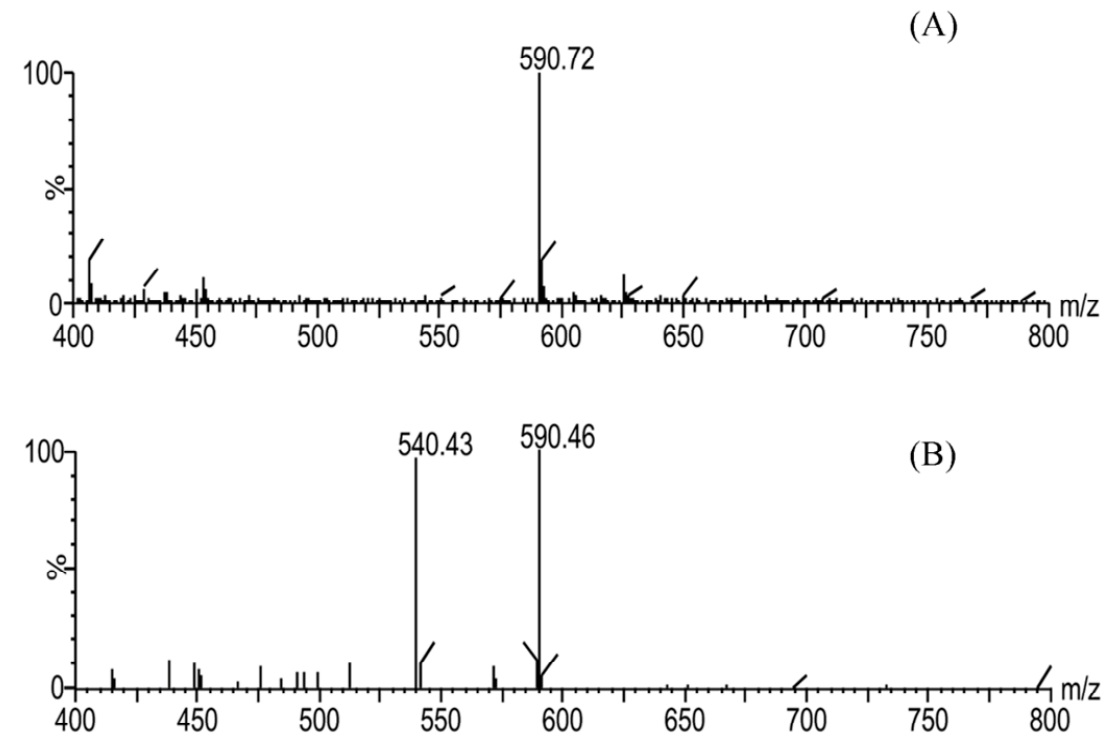

Figure 3. Full-scan (A) and MS/MS daughter scan (B) spectrograms of testosterone.

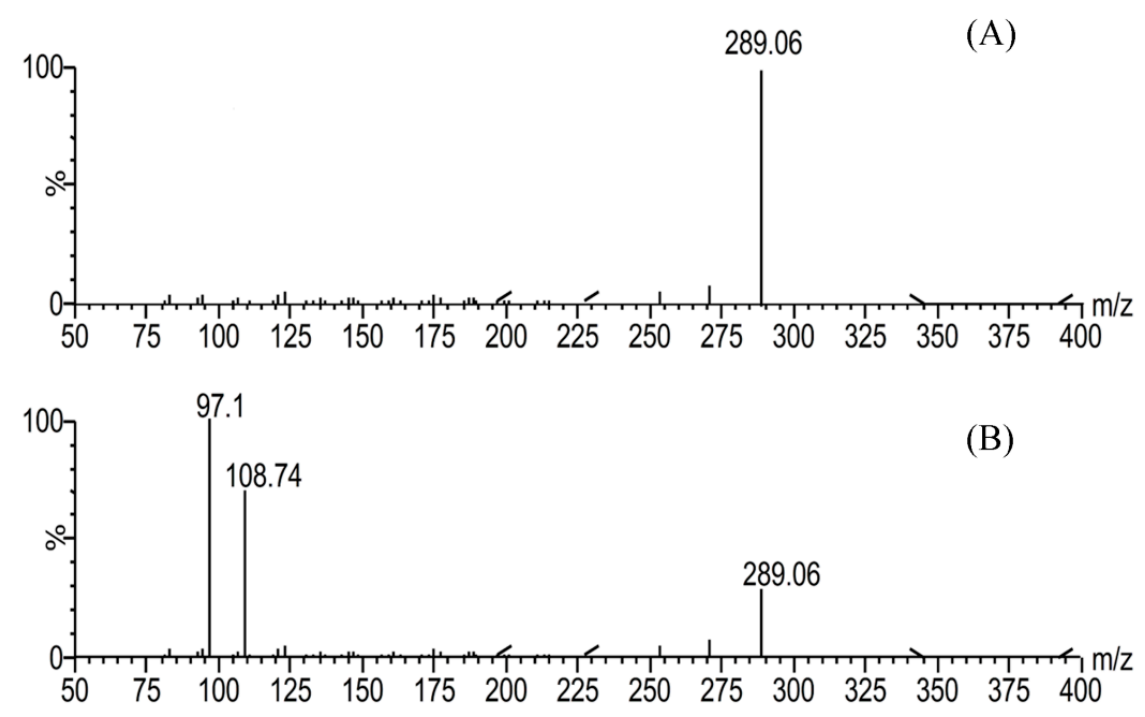

The chromatograms showed excellent peak shapes for BMA and testosterone at retention times of 2.8 and $3.6 \mathrm{~min}$, respectively, with no endogenous interference. The peak responses of blank plasma samples spiked with BMA and testosterone are shown in Figure 4. 
Figure 4. Chromatograms for BMA and testosterone in blank plasma (A); blank plasma spiked with BMA at $6 \mathrm{ng} / \mathrm{mL}$ and testosterone at $100 \mathrm{nM} \mathrm{(B);} \mathrm{plasma} \mathrm{sample} \mathrm{of} \mathrm{BMA} \mathrm{and}$ testosterone after administration of pure BMA $(\mathbf{C})$; and Wutou decoction $(\mathbf{D})$. The retention time of BMA and testosterone was 2.8 and $3.3 \mathrm{~min}$, which could be separated completely.

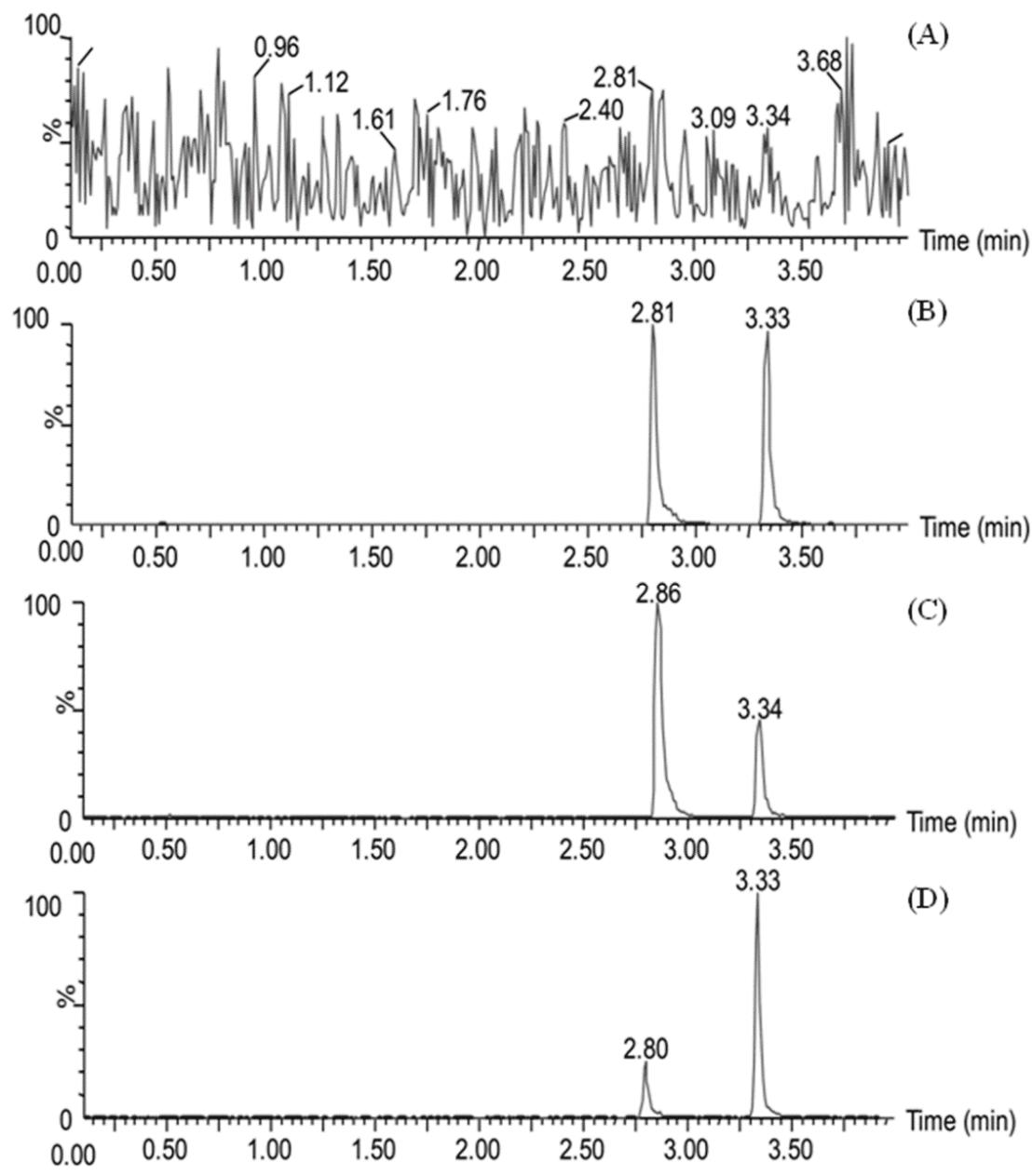

The calibration curve was investigated in the range of $0.3 \mathrm{ng} / \mathrm{mL}$ to $60 \mathrm{ng} / \mathrm{mL}$. All curves had correlation coefficients of $>0.990$. The lower limit of quantification (LLOQ) of BMA under the developed UPLC-MS/MS method was $0.3 \mathrm{ng} / \mathrm{mL}$, with a signal-to-noise ratio of $>10$.

The precision and accuracy of the method for detecting BMA were evaluated at three concentrations. The RSD $\%$ values for the intra-day and inter-day precision were smaller than $6.1 \%$ and $13.9 \%$, respectively. The RE\% values for the lowest concentration were $17.6 \%$ and smaller than $15.0 \%$ for the other two concentrations (see in Table 1).

Table 1. Accuracy and precision of BMA in rat plasma $(n=5)$.

\begin{tabular}{cccc}
\hline \multirow{2}{*}{$\begin{array}{c}\text { Concentrations } \\
(\mathbf{n g} / \mathbf{m L})\end{array}$} & $\begin{array}{c}\text { Accuracy } \\
\mathbf{( R E \% )}\end{array}$ & \multicolumn{2}{c}{ Precision (RSD\%) } \\
\cline { 3 - 4 } & 17.6 & Intra-day & Inter-day \\
\hline 1.2 & 12.7 & 6.1 & 13.9 \\
6 & 10.3 & 3.1 & 7.9 \\
30 & & & 4.7 \\
\hline
\end{tabular}


The extraction recovery values of BMA were $>85 \%$ at three concentrations. The matrix effects were over the range of $126.1 \%$ to $139.0 \%$ at low, middle and high concentrations, respectively, suggesting that significant ion suppression or enhancement did not occur at the expected retention times of the targeted ions (see in Table 2).

Table 2. Extraction recovery and matrix effect of BMA in rat plasma $(n=5)$.

\begin{tabular}{ccccc}
\hline \multirow{2}{*}{$\begin{array}{c}\text { Concentrations } \\
(\mathbf{n g} / \mathbf{m L})\end{array}$} & \multicolumn{2}{c}{ Extraction Recovery } & \multicolumn{2}{c}{ Matrix Effect } \\
\cline { 2 - 5 } & Mean $\mathbf{( \% )}$ & RSD (\%) & Mean (\%) & RSD (\%) \\
\hline 1.2 & 92.0 & 5.4 & 126.1 & 5.9 \\
6 & 92.9 & 9.3 & 139.0 & 2.3 \\
30 & 85.2 & 6.4 & 134.1 & 6.5 \\
\hline
\end{tabular}

The accuracy biases of BMA in the rat plasma samples ranged from $94.7 \%$ to $109.8 \%, 99.2 \%$ to $114.6 \%, 90.2 \%$ to $101.7 \%$ and $92.6 \%$ to $100.0 \%$ for the stability evaluation of short-term storage, long-term storage, three freeze-thaw cycles and auto-sampler storage, respectively (see in Table 3 ).

Table 3. Stability evaluation of BMA in rat plasma $(n=5)$.

\begin{tabular}{ccccccccc}
\hline \multirow{2}{*}{$\begin{array}{c}\text { Concentrations } \\
(\mathbf{n g} / \mathbf{m L})\end{array}$} & \multicolumn{2}{c}{$\begin{array}{c}\text { Short-Term } \\
\text { Storage }\end{array}$} & \multicolumn{2}{c}{$\begin{array}{c}\text { Long-Term } \\
\text { Storage }\end{array}$} & \multicolumn{2}{c}{$\begin{array}{c}\text { Three Freeze- } \\
\text { Thaw Cycles }\end{array}$} & \multicolumn{2}{c}{$\begin{array}{c}\text { Auto-Sampler } \\
\text { Stability }\end{array}$} \\
\cline { 2 - 9 } & $\begin{array}{c}\text { Accuracy } \\
(\%)\end{array}$ & $\mathbf{R S D} \%$ & $\begin{array}{c}\text { Accuracy } \\
\mathbf{( \% )}\end{array}$ & $\mathbf{R S D \%}$ & $\begin{array}{c}\text { Accuracy } \\
\mathbf{( \% )}\end{array}$ & RSD\% & $\begin{array}{c}\text { Accuracy } \\
\mathbf{( \% )}\end{array}$ & $\mathbf{R S D \%}$ \\
\hline 1.2 & 94.7 & 13.3 & 114.6 & 2.4 & 90.2 & 10.2 & 100.0 & 3.9 \\
6 & 109.8 & 5.1 & 110.3 & 3.3 & 100.8 & 4.0 & 106.4 & 8.3 \\
30 & 109.4 & 3.3 & 99.2 & 8.2 & 101.7 & 5.0 & 92.6 & 4.8 \\
\hline
\end{tabular}

\subsection{Content Determination of BMA in the Lyophilized Powder}

The content of BMA in the lyophilized powder was determined as follows. Lyophilized powder was dissolved in pure water to obtain the decoction solution. The solution was vortexed for 3 min and then centrifuged at 13,000 rpm for $30 \mathrm{~min}$. The supernatant was injected into the UPLC-MS/MS system for determination. The result showed that $0.92 \%$ of BMA was present in the lyophilized powder.

\subsection{Pharmacokinetic Study}

Although previous studies already investigated the pharmacokinetics of BMA [21,22], the present study is the first to compare the pharmacokinetic behavior of pure BMA and BMA as a marker compound of Wutou decoction in rats.

The developed UPLC-MS/MS method was used for the quantitation of plasma samples after oral administration of pure BMA $(5 \mathrm{mg} / \mathrm{kg})$ and Wutou decoction $(0.54 \mathrm{~g} / \mathrm{kg}$, equivalent to BMA $5 \mathrm{mg} / \mathrm{kg})$. As the predominant component of Wutou decoction, BMA exhibited the highest content in rat plasma after oral administration, which is in accordance with a previous study [21]. The plasma concentration versus time profile of BMA in the two rat groups is shown in Figure 5. 
Figure 5. Plasma concentration versus time profile of BMA after oral administration of BMA and Wutou decoction at a dosage of $5 \mathrm{mg} / \mathrm{kg}$ (BMA). Each point represents the mean and standard deviation (mean \pm SD) of five rats. BMA plasma concentrations at $240 \mathrm{~min}$ were approximately 0 after oral administration of Wutou decoction, and time points after that were deleted.

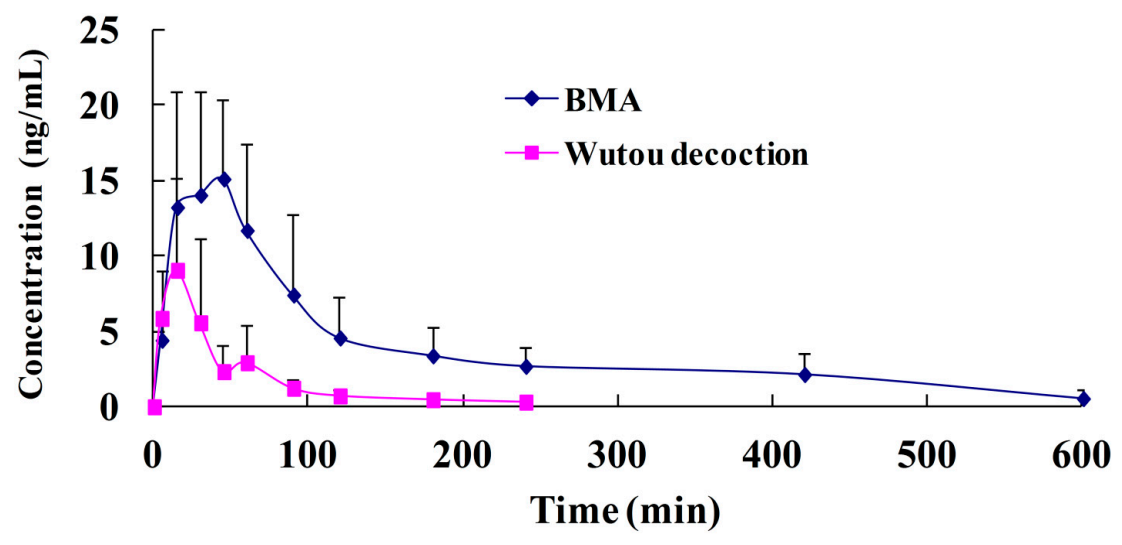

The pharmacokinetic parameters were expressed as the mean \pm SD. All parameters are shown in Table 4. After the oral administration of pure BMA and Wutou decoction, the plasma concentration-time curve produced a fast rising trend followed by a sharp decline with $T_{1 / 2}$ of $228.3 \pm 117.0$ min and $61.8 \pm 35.1 \mathrm{~min}$, respectively, until the levels fell below the detection limits. BMA was absorbed at a fast rate and reached a maximum concentration at $C_{\max }$ of $16.2 \pm 6.7 \mathrm{ng} / \mathrm{mL}$ and $10.0 \pm 5.8 \mathrm{ng} / \mathrm{mL}$ within $35.0 \pm 11.2 \mathrm{~min}$ and $13.0 \pm 4.5 \mathrm{~min}$ after pure BMA and Wutou decoction administration, respectively. The mean residence time (MRT) values for pure BMA were $155.0 \pm 33.2$ min, whereas that for Wutou decoction were $55.8 \pm 16.4 \mathrm{~min}$. The $\mathrm{AUC}_{(0-\mathrm{t})}$ values of BMA for pure BMA and Wutou decoction were $2247.4 \pm 1171.9$ and $447.8 \pm 292.2 \mathrm{ng} \cdot \mathrm{min} / \mathrm{mL}$, respectively, and the relative bioavailability of BMA after oral administration of Wutou decoction was $19.9 \%$. Furthermore, the results of the pharmacokinetic study indicated that the absorption and elimination of BMA after the administration of pure BMA and Wutou decoction were very fast, within $13.0 \mathrm{~min}$ to $35.0 \mathrm{~min}$ and $61.8 \mathrm{~min}$ to $228.3 \mathrm{~min}$, respectively.

Table 4. Pharmacokinetic parameters of BMA in Sprague-Dawley rat plasma $(n=5)$ after oral administration of BMA and Wutou decoction at $5 \mathrm{mg} / \mathrm{kg}(\mathrm{BMA}) . \mathrm{AUC}_{(0-\mathrm{t})}, T_{1 / 2}, C_{\max }$, $T_{\max }$, mean residence time (MRT) and relative bioavailability (RF) are shown in the table. Each value represents the mean and standard deviation (mean $\pm \mathrm{SD}$ ) of five rats.

\begin{tabular}{cccc}
\hline Parameters & Unit & BMA & Wutou Decoction \\
\hline $\mathrm{AUC}_{(0-\mathrm{t})}$ & $\mathrm{ng} \cdot \mathrm{min} / \mathrm{mL}$ & $2,247.4 \pm 1,171.9$ & $447.8 \pm 292.2 *$ \\
$T_{1 / 2}$ & $\mathrm{~min}$ & $228.3 \pm 117.0$ & $61.8 \pm 35.1$ \\
$C_{\max }$ & $\mathrm{ng} / \mathrm{mL}$ & $16.2 \pm 6.7$ & $10.0 \pm 5.8$ \\
$T_{\max }$ & $\min$ & $35.0 \pm 11.2$ & $13.0 \pm 4.5^{*}$ \\
$\mathrm{MRT}$ & $\min$ & $155.0 \pm 33.2$ & $55.8 \pm 16.4 *$ \\
$\mathrm{RF}$ & $\%$ & - & 19.9 \\
\hline \multicolumn{4}{c}{$* 0.05$ between two groups. }
\end{tabular}


Notably, the pharmacokinetic process of BMA in Wutou decoction was significantly different compared with that in pure BMA (ANOVA, $p<0.05$ ). AUC $(0-\mathrm{t}), T_{1 / 2}$ and MRT decreased, suggesting that other complex ingredients in Wutou decoction might have an influence on the pharmacokinetics of BMA, which possibly demonstrated shorter retention time in vivo and lower distribution in the target organs, leading potentially to toxicity attenuation.

Numerous comparative pharmacokinetic studies have been reported on the absorption of effective components enhanced after combination with other herbs, some of which results from the competitive inhibition between components in herbs on the elimination via efflux transporters or the metabolism via drug metabolizing enzymes, such as CYP3A (microsomal cytochrome P450) [18]. The possible mechanism for the decrease in pharmacokinetic parameters is that the drug efflux transporters and CYP3A are distributed abundantly in the enterocytes of the gastrointestinal tract, which is the crucial place for the absorption of orally administered drugs. Therefore, the excretion by intestinal efflux transporters or metabolism by CYP3A might reduce drug absorption.

Although the exact mechanisms accounting for the different pharmacokinetic behaviors of BMA after pure BMA and Wutou decoction administration are not clear, different herb-herb interactions in Wutou decoction might be one possible explanation. The compounds in Wutou decoction might play an important role in affecting the elimination of BMA, influencing several links of ADME (absorption, distribution, metabolism and elimination) of BMA in rats, which leads to the decreased AUC and $T_{1 / 2}$. For example, the efflux transporter, MRP2 (multi-drug resistance associate protein 2), was demonstrated to be involved in the efflux of BMA [6]. The diminished $\mathrm{AUC}_{(0-\mathrm{t})}$ might suggest that other complex ingredients in Wutou decoction reduced the absorption and bioavailability of BMA via induction of MRP2 efflux.

Furthermore, it was also reported that drug metabolizing enzymes, including CYP3A (microsomal cytochrome P450), played a significant role in the elimination of some herbs in vitro [23,24]. Meanwhile, BMA could be also metabolized by CYP3A [15]. Accelerating the metabolic elimination of BMA would reduce the oral bioavailability of BMA. Thus, another factor that may contribute to the faster elimination of BMA in Wutou decoction is metabolic induction by the other coexisting constituents. $T_{1 / 2}$ and MRT decreased, which indicated that some components in Wutou decoction might induce the metabolism of CYP3A, thereby shortening the retention time of BMA in vivo, which might contributed to the toxicity reduction. Among all of the components of Wutou decoction, diammonium glycyrrhizinate reportedly has a positive effect on the toxicity reduction of aconitum alkaloids $[25,26]$.

Thus, the faster elimination of BMA in rats after Wutou decoction administration is likely to be attributed to efflux through MRP2 or metabolism by CYP3A4/5 [6]. Some in vivo herb-herb interactions between the complex ingredients of Wutou decoction might exist to decrease the AUC and MRT of BMA via the enhanced expression of MRP2 or CYP3A. However, direct research on the induced expression of CYP3A or MRP2 by the complex ingredients in Wutou decoction is still limited, leading to more studies on a deeper level. 


\section{Experimental Section}

\subsection{Chemicals and Reagents}

BMA ( $\geq 98 \%$ purity) was obtained from the National Institute for the Control of Pharmaceutical and Biological Products (Beijing, China). Testosterone ( $\geq 98 \%$ purity) was purchased from Nacalai Tesque (Kyoto, Japan). Radix Aconiti, Herba Ephedrae, Radix Paeoniae Alba, Radix Astragali and Radix Glycyrrhizae (identified by the Sichuan Institute for Food and Drug Control) were purchased from Dongguan China Herbal Medicine Co., Ltd. (Dongguan, China). A voucher specimen was deposited at the Laboratory of Pharmaceutics, School of Pharmaceutical Sciences, Southern Medical University (Guangzhou, China). All other chemicals were of analytical grade.

\subsection{Animals}

Male Sprague-Dawley rats weighing between 230 and $280 \mathrm{~g}$ were supplied by the Laboratory Animal Center of Southern Medicine University (license: SCXK, Guangdong, 2006-0015) (Guangzhou, China). The rats were housed four per cage in a unidirectional airflow room under controlled temperature $\left(20{ }^{\circ} \mathrm{C}\right.$ to $\left.24{ }^{\circ} \mathrm{C}\right)$, relative humidity $(40 \%$ to $70 \%)$ and a 12 -h light/dark cycle. The animal experimental protocol was approved by the ethics committee of Southern Medicine University (No: 2011-0015, Date: 24 October 2012). All animal studies were carried out according to the guide for care and use of laboratory animals. The rats were fasted, but allowed free access to water for at least $12 \mathrm{~h}$ before the experiment.

\subsection{Instruments and Conditions}

The UPLC-MS/MS system (Waters, Milford, MA, USA) consisted of a binary solvent manager, a column compartment, an auto-sampler manager and a single quadrupole mass spectrometer. All data acquisition was performed with Waters Masslynx V4.1 software.

The liquid chromatography separation of plasma samples was carried out on an Acquity UPLC BEH C18 column $(1.7 \mu \mathrm{m} ; 2.1 \mathrm{~mm} \times 50 \mathrm{~mm})$ at $40{ }^{\circ} \mathrm{C}$. Two micromolar ammonium acetate (A) and $100 \%$ acetonitrile (B) were used as the mobile phase. The flow rate was set at $0.3 \mathrm{~mL} / \mathrm{min}$. The gradients used for the elution were $0 \mathrm{~min}, 95 \% \mathrm{~A} ; 0 \mathrm{~min}$ to $1.2 \mathrm{~min}, 95 \%$ to $88 \% \mathrm{~A} ; 1.2 \mathrm{~min}$ to $3 \mathrm{~min}$, $88 \%$ to $20 \% \mathrm{~A}$; and $3 \mathrm{~min}$ to $4 \mathrm{~min}, 20 \%$ to $95 \% \mathrm{~A}$. The injection volume was $5 \mu \mathrm{L}$.

Electro spray ionization in the positive mode was used to detect BMA in the plasma samples. The MS tune parameters were as follows: capillary voltage, $3.0 \mathrm{kV}$; cone voltage, $30 \mathrm{~V}$; ion source temperature, $120{ }^{\circ} \mathrm{C}$; desolvation temperature, $350{ }^{\circ} \mathrm{C}$; desolvation gas flow, $650 \mathrm{~L} / \mathrm{h}$; and cone gas flow, $50 \mathrm{~L} / \mathrm{h}$. The mass spectrometer was operated in the multiple reaction monitoring (MRM) mode.

\subsection{Preparation of Wutou Decoction Lyophilized Powder}

Radix Aconiti (6 g) was added to $600 \mathrm{~mL}$ of boiling pure water and then boiled for 30 min to extract its active constituents. A mixture of Herba Ephedrae (9 g), Radix Paeoniae Alba (9 g), Radix Astragali (9 g) and Radix Glycyrrhizae ( $9 \mathrm{~g}$ ) was added and boiled for $30 \mathrm{~min}$. The solution was filtered and concentrated to obtain Wutou decoction. The decoction was concentrated to $100 \mathrm{~mL}$ using a rotary 
evaporator and then stored at $-20{ }^{\circ} \mathrm{C}$ overnight for freezing. The frozen decoctions were freeze dried by a lyophilizer to obtain the lyophilized powder form of the decoction. The content of BMA in the lyophilized powder was quantified by UPLC-MS/MS the day before the pharmacokinetic experiment.

\subsection{Preparation of Stock Solutions}

A stock solution of BMA was prepared in methanol at a concentration of $5 \mathrm{mM}$, and the working solution of BMA was diluted with methanol. The testosterone stock solution was prepared in acetonitrile at a concentration of $20 \mathrm{mM}$, and the working solution of testosterone was diluted to $1000 \mathrm{nM}$ with acetonitrile. All stock solutions were stored at $-20{ }^{\circ} \mathrm{C}$ prior to use.

\subsection{Biosample Collection}

Pure BMA and Wutou decoction lyophilized powder was dissolved in $0.9 \%$ saline to obtain a concentration of $0.25 \mathrm{mg} / \mathrm{mL}$ (BMA). Ten rats were randomly divided into two groups. Pure BMA $(5 \mathrm{mg} / \mathrm{kg})$ and Wutou decoction $(0.54 \mathrm{~g} / \mathrm{kg}$, equivalent to $5 \mathrm{mg} / \mathrm{kg}$ BMA) were orally administered to them, respectively. Serial blood samples $(500 \mu \mathrm{L})$ were taken from the orbital sinus venous plexus at 0 , $5,15,30,45,60,90,120,180,240,420$ and $600 \mathrm{~min}$ after gavage dosing. Each collected blood sample was centrifuged at $8000 \mathrm{rpm}$ for $8 \mathrm{~min}$. The plasma fractions were transferred into a disposable tube and then frozen at $-80{ }^{\circ} \mathrm{C}$ until analysis.

\subsection{Biosample Preparation}

To detect BMA in the rat plasma, $80 \mu \mathrm{L}$ of plasma was mixed with $320 \mu \mathrm{L}$ methanol containing $100 \mathrm{nM}$ testosterone. The mixture was centrifuged at 13,000 rpm for $30 \mathrm{~min}$. A total of $300 \mu \mathrm{L}$ of the supernatant was transferred to a disposable tube and then evaporated to dryness under a stream of nitrogen at room temperature. The residue was reconstituted with $100 \mu \mathrm{L}$ of methanol-water $(\mathrm{v}: \mathrm{v}=1: 1)$ and then injected into the UPLC-MS/MS system for analysis.

\subsection{Method Validation}

The specificity of the method was evaluated using blank plasma samples. Blank rat plasma was spiked with the working solutions of BMA $(6 \mathrm{ng} / \mathrm{mL})$ and testosterone $(100 \mathrm{nM})$. The linearity of BMA in rat plasma was evaluated with the calibration curve based on the UPLC-MS/MS analysis of blank plasma spiked with different concentrations of BMA. This curve was obtained by plotting the peak area ratios versus the different concentrations of BMA. The LLOQ was defined based on the minimum concentration with a signal-to-noise ratio of $\geq 10$. The precision and accuracy were evaluated by analyzing quality control (QC) samples with different concentrations. Intra-day and inter-day precision and accuracy were evaluated for 3 consecutive days with five replicates at each concentration per day.

The recoveries were determined by comparing the peak areas from the extracted samples with those from post-extracted blank plasma spiked with the analytes at the same concentration. The matrix effect was measured by comparing the peak areas of analytes added into the post-extracted blank with analytes dissolved in the matrix component-free reconstitution solvent. The stability of BMA in rat 
plasma was evaluated at room temperature for $12 \mathrm{~h}$, after storage at $-80{ }^{\circ} \mathrm{C}$ for 15 days, after three freeze-thaw cycles or in an auto-sampler for $12 \mathrm{~h}$, respectively.

\subsection{Data Analysis}

The PK parameters were determined using the standard non-compartmental method and calculated using WinNonlin 5.2 (Pharsight, Mountain View, CA, USA). The $C_{\max }$ and the corresponding $T_{\max }$ were directly obtained from the raw data. The AUC to the last measurable concentration $\left(\mathrm{AUC}_{(0-\mathrm{t})}\right)$ was calculated using the linear trapezoidal method. The relative bioavailability of BMA after oral administration of Wutou decoction was calculated as $\mathrm{AUC}_{(\text {decoction })} / \mathrm{AUC}_{(\mathrm{BMA})} \times 100 \%$. An independent-sample $t$-test was performed twice to evaluate the differences of pharmacokinetic parameters between the two groups.

\section{Conclusions}

To the best of our knowledge, this study is the first to compare the pharmacokinetics of BMA after oral administration of pure BMA and Wutou decoction. The absorption of BMA in Wutou decoction was significantly reduced due to faster elimination, in comparison with that of pure BMA administration, which might contribute to toxicity reduction. Better understanding of the interactions between BMA and the coexisting ingredients in the Wutou decoction is still needed for clinical application of Wutou decoction.

\section{Acknowledgments}

We would like to thank the Sichuan Institute for Food and Drug Control for the help of the identification of the plant materials. This work was supported in part by the Key International Joint Research Project of National Natural Science Foundation of China (Grant No. 81120108025), the Science and Information Technology of Guangzhou (Grant No. 2011Y1-00017-6) and the Fund of the Natural Science Foundation of Guangdong Province (Grant No. S2013040012007).

\section{Author Contributions}

Conceived of and designed the experiments: Zhong-Qiu Liu, Pei-Min Dai, Ling Ye. Performed the experiments: Pei-Min Dai, Shan Zeng, Ying Wang, Zhi-Jie Zheng, Qiang Li, Lin-Lin Lu. Analyzed the data: Pei-Min Dai, Shan Zeng, Ying Wang, Zhi-Jie Zheng. Wrote the paper: Zhong-Qiu Liu, Pei-Min Dai, Ling Ye, Ying Wang.

\section{Conflicts of Interest}

The authors declare no conflict of interest.

\section{References}

1. Fraenkel, L.; Cunningham, M. High disease activity may not be sufficient to escalate care. Arthrit. Care Res. 2014, 66, 197-203. 
2. Wisniacki, N.; Amaravadi, L.; Galluppi, G.R.; Zheng, T.S.; Zhang, R.; Kong, J.; Burkly, L.C. Safety, tolerability, pharmacokinetics, and pharmacodynamics of anti-TWEAK monoclonal antibody in patients with rheumatoid arthritis. Clin. Ther. 2013, 35, 1137-1149.

3. Van Landeghem, A.A.; de Letter, E.A.; Lambert, W.E.; van Peteghem, C.H.; Piette, M.H. Aconitine involvement in an unusual homicide case. Int. J. Legal Med. 2007, 121, 214-219.

4. Singh, S.; Fadnis, P.P.; Sharma, B.K. Aconite poisoning. J. Assoc. Phys. India 1986, 34, 825-826.

5. Bisset, N.G. Arrow poisons in China. Part II. Aconitum-Botany, chemistry, and pharmacology. J. Ethnopharmacol. 1981, 4, 247-336.

6. Ye, L.; Yang, X.; Yang, Z.; Gao, S.; Yin, T.; Liu, W.; Wang, F.; Hu, M.; Liu, Z. The role of efflux transporters on the transport of highly toxic aconitine, mesaconitine, hypaconitine, and their hydrolysates, as determined in cultured Caco-2 and transfected MDCKII cells. Toxicol. Lett. 2013, 216, 86-99.

7. Singhuber, J.; Zhu, M.; Prinz, S.; Kopp, B. Aconitum in traditional Chinese medicine: A valuable drug or an unpredictable risk? J. Ethnopharmacol. 2009, 126, 18-30.

8. Elliott, S.P. A case of fatal poisoning with the aconite plant: Quantitative analysis in biological fluid. Sci. Justice 2002, 42, 111-115.

9. Pullela, R.; Young, L.; Gallagher, B.; Avis, S.P.; Randell, E.W. A case of fatal aconitine poisoning by Monkshood ingestion. J. Forensic Sci. 2008, 53, 491-494.

10. Jiang, Z.H.; Xie, Y.; Zhou, H.; Wang, J.R.; Liu, Z.Q.; Wong, Y.F.; Cai, X.; Xu, H.X.; Liu, L. Quantification of aconitum alkaloids in aconite roots by a modified RP-HPLC method. Phytochem. Anal. 2005, 16, 415-421.

11. Suzuki, Y.; Hayakawa, Y.; Oyama, T.; Isono, T.; Ohmiya, Y.; Ikeda, Y.; Asami, A.; Noguchi, M. Analgesic effect of benzoylmesaconine. Nihon Yakurigaku Zasshi 1993, 102, 399-404.

12. Suzuki, Y.; Oyama, T.; Ishige, A.; Isono, T.; Asami, A.; Ikeda, Y.; Noguchi, M.; Omiya, Y. Antinociceptive mechanism of the aconitine alkaloids mesaconitine and benzoylmesaconine. Planta Med. 1994, 60, 391-394.

13. Kobayashi, M.; Mori, K.; Kobayashi, H.; Pollard, R.B.; Suzuki, F. The regulation of burn-associated infections with herpes simplex virus type 1 or Candida albicans by a non-toxic aconitine-hydrolysate, benzoylmesaconine. Part 1: Antiviral and anti-fungal activities in thermally injured mice. Immunol. Cell Biol. 1998, 76, 202-208.

14. Kobayashi, M.; Takahashi, H.; Herndon, D.N.; Pollard, R.B.; Suzuki, F. Therapeutic effects of IL-12 combined with benzoylmesaconine, a non-toxic aconitine-hydrolysate, against herpes simplex virus type 1 infection in mice following thermal injury. Burns 2003, 29, 37-42.

15. Ye, L.; Yang, X.S.; Lu, L.L.; Chen, W.Y.; Zeng, S.; Yan, T.M.; Dong, L.N.; Peng, X.J.; Shi, J.; Liu, Z.Q. Monoester-diterpene aconitum alkaloid metabolism in human liver microsomes: Predominant role of CYP3A4 and CYP3A5. Evid.-Based Complement. Altern. Med. 2013, 2013, doi:10.1155/2013/941093.

16. Ye, L.; Gao, S.; Feng, Q.; Liu, W.; Yang, Z.; Hu, M.; Liu, Z. Development and validation of a highly sensitive UPLC-MS/MS method for simultaneous determination of aconitine, mesaconitine, hypaconitine, and five of their metabolites in rat blood and its application to a pharmacokinetics study of aconitine, mesaconitine, and hypaconitine. Xenobiotica 2012, 42, 518-525. 
17. Zhang, F.; Tang, M.H.; Chen, L.J.; Li, R.; Wang, X.H.; Duan, J.G.; Zhao, X.; Wei, Y.Q. Simultaneous quantitation of aconitine, mesaconitine, hypaconitine, benzoylaconine, benzoylmesaconine and benzoylhypaconine in human plasma by liquid chromatography-tandem mass spectrometry and pharmacokinetics evaluation of "SHEN-FU" injectable powder. J. Chromatogr. B 2008, 873, 173-179.

18. Liu, X.; Li, H.; Song, X.; Qin, K.; Guo, H.; Wu, L.; Cai, H.; Cai, B. Comparative pharmacokinetics studies of benzoylhypaconine, benzoylmesaconine, benzoylaconine and hypaconitine in rats by LC-MS method after administration of Radix Aconiti Lateralis Praeparata extract and Dahuang Fuzi Decoction. Biomed. Chromatogr. 2014, 28, 966-973.

19. Peng, W.W.; Li, W.; Li, J.S.; Cui, X.B.; Zhang, Y.X.; Yang, G.M.; Wen, H.M.; Cai, B.C. The effects of Rhizoma Zingiberis on pharmacokinetics of six Aconitum alkaloids in herb couple of Radix Aconiti Lateralis-Rhizoma Zingiberis. J. Ethnopharmacol. 2013, 148, 579-586.

20. Usui, K.; Hayashizaki, Y.; Hashiyada, M.; Nakano, A.; Funayama, M. Simultaneous determination of 11 aconitum alkaloids in human serum and urine using liquid chromatography-tandem mass spectrometry. Legal Med. 2012, 14, 126-133.

21. Tang, L.; Gong, Y.; Lv, C.; Ye, L.; Liu, L.; Liu, Z. Pharmacokinetics of aconitine as the targeted marker of Fuzi (Aconitum carmichaeli) following single and multiple oral administrations of Fuzi extracts in rat by UPLC/MS/MS. J. Ethnopharmacol. 2012, 141, 736-741.

22. Song, J.Z.; Han, Q.B.; Qiao, C.F.; But, P.P.; Xu, H.X. Development and validation of a rapid capillary zone electrophoresis method for the determination of aconite alkaloids in aconite roots. Phytochem. Anal. 2010, 21, 137-143.

23. Izzo, A.A.; Ernst, E. Interactions between herbal medicines and prescribed drugs: An updated systematic review. Drugs 2009, 69, 1777-1798.

24. Saxena, A.; Tripathi, K.P.; Roy, S.; Khan, F.; Sharma, A. Pharmacovigilance: Effects of herbal components on human drugs interactions involving cytochrome P450. Bioinformation 2008, 3, 198-204.

25. Peter, K.; Schinnerl, J.; Felsinger, S.; Brecker, L.; Bauer, R.; Breiteneder, H.; Xu, R.; Ma, Y. A novel concept for detoxification: Complexation between aconitine and liquiritin in a Chinese herbal formula ('Sini Tang'). J. Ethnopharmacol. 2013, 149, 562-569.

26. Chen, L.; Yang, J.; Davey, A.K.; Chen, Y.X.; Wang, J.P.; Liu, X.Q. Effects of diammonium glycyrrhizinate on the pharmacokinetics of aconitine in rats and the potential mechanism. Xenobiotica 2009, 39, 955-963.

Sample Availability: Sample of the compound BMA is available from the authors.

(C) 2014 by the authors; licensee MDPI, Basel, Switzerland. This article is an open access article distributed under the terms and conditions of the Creative Commons Attribution license (http://creativecommons.org/licenses/by/4.0/). 